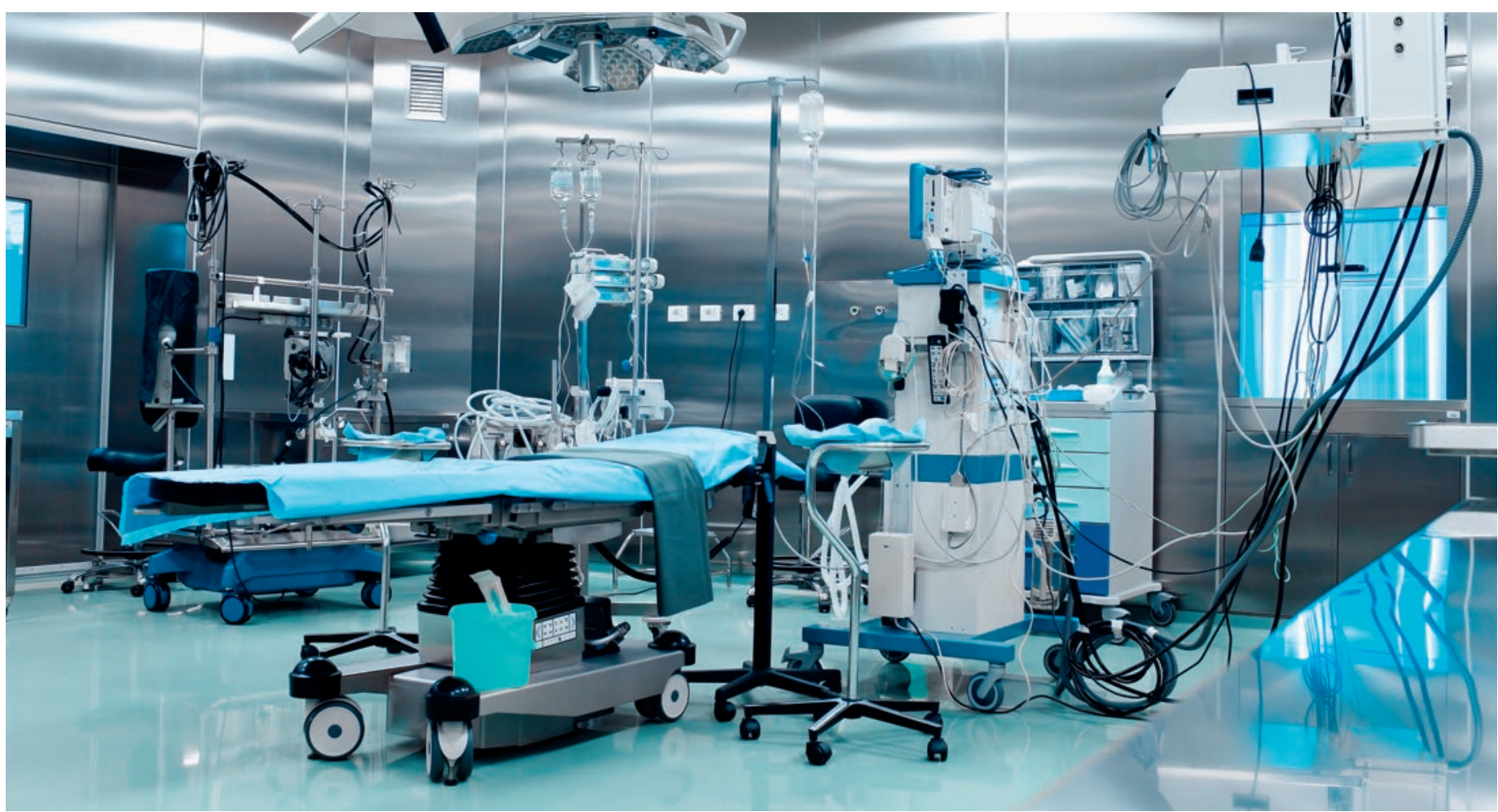

\title{
Verschiebung planbarer Eingriffe bei Ressourcenknappheit
}

\author{
Bernhard Rütsche ${ }^{a}$, Walter Fellmanna ${ }^{a}$ Regina E. Aebi-Müllera, Guido Schüpferb
}

${ }^{a}$ Prof. Dr. iur., Mitglied der Juristischen Fakultät, Universität Luzern; ${ }^{b}$ Dr. med., Klinik für Anästhesie, Luzerner Kantonsspital

Während der Covid-19-Pandemie kam es an diversen Spitälern zur Verschiebung von nicht dringlichen, planbaren Eingriffen. Ob und inwiefern diese Reallokation von Ressourcen gerechtfertigt ist, steht jedoch zur Debatte.

Zu Beginn der Covid-19-Pandemie erarbeiteten die Schweizerische Akademie der Medizinischen Wissenschaften (SAMW) und die Schweizerische Gesellschaft für Intensivmedizin (SGI) Richtlinien zur Triage von intensivmedizinischen Behandlungen bei Ressourcenknappheit. In der ersten Welle der Pandemie reichten die Verschiebung von planbaren Eingriffen, die Erhöhung der intensivmedizinischen Kapazitäten und die Massnahmen des Bundes aus, um in der Schweiz eine Ressourcenknappheit abzuwenden. Obwohl die Triagekriterien von SAMW und SGI offiziell nicht zur Anwendung kamen, muss davon ausgegangen werden, dass schon in der ersten Pandemiewelle implizit zugunsten von Covid-Patientinnen und -Patienten priorisiert wurde: Beansprucht nämlich ein Covid-Patient während mehr als 14 Tagen ein Intensivbett, so steht diese Ressource für zahlreiche Herz- oder Krebseingriffe nicht zur Verfügung, die zwar grundsätzlich zeitlich planbar sind, nach Verstreichen einer gewissen Zeitspanne aber trotzdem dringlich werden. Die $\mathrm{Zu}-$ rücksetzung solcher Behandlungen wiegt umso schwerer, als die betroffenen Patientinnen und Patienten bis zur Stabilisierung ihrer Vitalfunktionen meist nur für ein bis zwei Tage einen Intensivplatz bräuchten.

\section{Rettung möglichst vieler Leben}

Im Herbst 2020 haben zahlreiche Spitäler über mehrere Monate hinweg auch ihre Operationskapazitäten gezielt und deutlich reduziert (insbesondere durch 
Schliessung von Operationssälen) und die gewonnenen Ressourcen, u.a. Anästhesiepersonal, zugunsten von Covid-Erkrankten eingesetzt. Von der Einschränkung der Operationskapazitäten war auch eine Vielzahl von Hospitalisierten betroffen, deren Eingriff ohne Intensivplatz hätte durchgeführt werden können. Es ist davon auszugehen, dass während der zweiten Pandemiewelle bis Februar 2021 mehr als 20000 planbare Eingriffe verschoben wurden. Viele dieser Eingriffe lassen sich aber nicht unbegrenzt hinauszögern. Die mehrwöchige oder -monatige Verschiebung

Mit Überschreitung des massgeblichen Zeitfensters wird eine an sich planbare Operation zu einer dringlichen Operation.

einer planbaren Operation kann zu einer Verschlechterung der Prognose, zu irreversiblen Gesundheitsschäden und sogar zu vorzeitigem Tod führen. Nicht ohne Grund ist die Wartezeit für planbare Eingriffe ein Indikator für die Qualität eines Gesundheitswesens. Mit Überschreitung des massgeblichen Zeitfensters wird eine an sich planbare Operation zu einer dringlichen Operation. Die Verschiebung eines planbaren Eingriffs ist letztlich nichts anderes als die vorläufige Verweigerung eines medizinisch gebotenen Eingriffs. Patientinnen und Patienten, deren Überlebensprognose oder Chancen auf vollständige Heilung bei adäquater und zeitnaher Behandlung gut gewesen wären, wurden somit sozusagen Opfer der ausgedünnten Personalbestände und anderer verschobener Ressourcen. Die in Medien und Politik verwendete Umschreibung einer «Verschiebung elektiver Eingriffe» [1] während der Covid-19-Pandemie verharmlost diese Fakten, weil sie zur Annahme verleitet, dass lediglich frei wählbare Eingriffe betroffen waren, deren Verschiebung für die Patientinnen und Patienten keine gesundheitlichen Folgen hatte.

Fest steht, dass manche Spitäler während der Pandemie dringliche intensivmedizinische Behandlungen (insbesondere von Covid-Erkrankten) gegenüber dringlichen nicht-intensivmedizinischen Behandlungen priorisiert haben. Diese Priorisierung erfolgte durch Ressourcenreallokation und somit auf institutioneller Ebene, und nicht in Einzelfällen in Form einer Bevorzugung bestimmter Patientinnen und Patienten. Als Rechtfertigung der Erweiterung von Intensivkapazitäten mag die Rettung des Lebens möglichst vieler unmittelbar mit dem Tod bedrohter Menschen dienen. Der Entscheid, der unmittelbaren Lebensrettung möglichst vieler Personen oberste Priorität einzuräumen, wendet jedoch den Blick vom Alter und Gesundheitszustand der intensivmedizinisch behandelten Patien- tinnen und Patienten ab und lässt offen, wie viele Lebensjahre und wie viel Lebensqualität durch die Verweigerung planbarer medizinischer Behandlungen ausserhalb der Intensivstationen aufgegeben werden. Ob mit der Reallokation der Spitalressourcen der medizinische Gesamtnutzen maximiert wurde, bleibt somit ungeklärt.

\section{Gesetzliche Legitimation erforderlich}

Aus Sicht der Grundrechte lässt sich der Vorrang unmittelbarer Lebensrettung vor der Rettung möglichst vieler Lebensjahre und der Erhaltung bzw. Verbesserung der Lebensqualität möglichst vieler Patientinnen und Patienten nicht beanstanden. Dies jedenfalls dann nicht, wenn man von einer Grundrechtstheorie ausgeht, nach der sich das Gewicht von Grundrechten nicht nach der Menge der Betroffenen, sondern nach der Intensität - und damit auch nach der Unmittelbarkeit - der Betroffenheit bestimmt (Nicht-Quantifizierbarkeit von Rechten). Auch der Pandemieplan des Bundes von 2018 gibt der «Rettung möglichst vieler lebensbedrohlich erkrankter Menschen» [2] oberste Priorität. Im Übrigen gehen die Richtlinien von SAMW und SGI zur Triage von intensivmedizinischen Behandlungen bei Ressourcenknappheit implizit ebenfalls vom Vorrang der unmittelbaren Lebensrettung aus, indem sie zur Vermeidung von Knappheitssitua-

Wird eine planbare Operation verschoben, kann dies die Prognose verschlechtern, zu irreversiblen Gesundheitsschäden und sogar zum vorzeitigen Tod führen.

tionen unter anderem die Erhöhung der Intensivplätze mittels Einstellung planbarer Eingriffe verlangen. Ob in einer akuten Knappheitssituation wie der Covid19-Pandemie das Kriterium der unmittelbaren Lebensrettung generell über das Kriterium der medizinischen Behandlungsaussichten (medizinischer Nutzen) gestellt werden soll, ist jedoch eine fundamentale Wertentscheidung. Diese Entscheidung sollte nicht allein in einem behördlichen Pandemieplan oder einer nicht-staatlichen Richtlinie getroffen, sondern öffentlich diskutiert und auf eine demokratische Legitimationsbasis gestellt werden.

\section{Spitalplanung hat sich (auch) auf Krisen- situationen auszurichten}

Grundsätzlich sind Spitäler, die auf der Spitalliste eines Kantons figurieren, entsprechend ihren Kapazitäten verpflichtet, für Kantonseinwohnende eine Aufnahme 
zu gewährleisten. Allerdings fehlen in der Spitalplanung und den darauf basierenden Leistungsaufträgen der Kantone an ihre Spitäler Mindestvorgaben zur Gewährleistung der Versorgung im Krisenfall. Primäres Ziel der Spitalplanung ist vielmehr der Abbau von sogenannten Überkapazitäten.

\section{Die Verantwortung für die Verweigerung} planbarer medizinischer Eingriffe liegt bei den Kantonen und damit der Politik.

Mit Blick auf den - verfassungsrechtlich haltbaren Vorrang der unmittelbaren Lebensrettung kann den Spitälern kein Vorwurf gemacht werden, dass sie aufgrund fehlender Kapazitäten für die Bewältigung des voraussichtlichen Behandlungsbedarfs Ressourcen zugunsten von Covid-19-Erkrankten realloziert haben. Die Verantwortung für die Verweigerung planbarer medizinischer Eingriffe verlagert sich damit auf die politische Ebene: In der Pflicht stehen vor allem die Kantone als Verantwortliche für die bedarfsgerechte Spitalversorgungsplanung. Mittels Planung und Vorgabe von Mindestkapazitäten im stationären Bereich oder mit der Einrichtung eines funktionierenden Systems für die (interkantonale) Verteilung von Patientinnen und Patienten sollten diese, soweit möglich, dafür sorgen, dass zeitrelevante Eingriffe auch in Krisensituationen wie einer Pandemie vorgenommen werden können. Es kann nicht ausgeschlossen werden, dass das Unterlassen solcher Massnahmen zur Gewährleistung der Spitalversorgung für planbare Eingriffe im Einzelfall Staatshaftungsansprüche geschädigter Patientinnen und Patienten gegenüber den verantwortlichen Kantonen begründet.

\section{Treue- und Sorgfaltspflicht}

Das Arzt-Patienten-Verhältnis ist von der Treuepflicht des Beauftragten geprägt. Der Arzt oder die Ärztin ist verpflichtet, die Interessen der Patientinnen und Patienten umfassend zu wahren. Sie müssen den Inter-

Das Selbstbestimmungsrecht der Patientin und des Patienten ist zentral - sie sollten ein Gegenüber auf Augenhöhe sein.

essen der Erkrankten gegenüber allen anderen Interessen stets den Vorrang einräumen - selbst gegenüber den eigenen. Im Zuge dieser Interessenwahrungspflicht hat die Ärztin oder der Arzt für eine sorgfältige, auf die Wiederherstellung der Gesundheit ausgerichtete Behandlung nach den Regeln der medizinischen
Kunst einzustehen. Überdies unterliegen sie einer umfassenden Aufklärungs- und Benachrichtigungspflicht. Zentral ist in diesem Zusammenhang das Selbstbestimmungsrecht der Patientin und des Patienten. Diese sollen idealerweise und gerade bei planbaren Eingriffen ein Gegenüber auf Augenhöhe sein und - nach gebührender Information - selbst entscheiden, ob, wann und von welchem Gesundheitsdienstleistungserbringer sie behandelt werden wollen.

Wenn ein Spital wegen zahlreicher Covid-Erkrankter Operationen absagen oder verschieben muss, kann dem behandelnden Arzt oder der behandelnden Ärztin deswegen kein Vorwurf gemacht werden. Eine Ressourcenknappheit entbindet die Behandelnden aber nicht von ihren Pflichten. Sie müssen daher die Betroffenen von sich aus über die Gründe für die Verschiebung, den voraussichtlichen (verzögerten) Termin der Operation, die konkreten gesundheitlichen Folgen bzw. Risiken der Verschiebung sowie über das gebotene Verhalten in der Wartezeit (z.B. in Bezug auf die

\section{In der Spitalplanung fehlen Mindest-} vorgaben zur Gewährleistung der Versorgung im Krisenfall.

Medikation) informieren. Zudem soll über mögliche alternative Behandlungen respektive Anbieter aufgeklärt werden. Ist der geplante Eingriff beispielsweise an einem anderen Spital - auch einem ausserkantonalen - ohne Aufschub möglich, so müssen Patientinnen und Patienten darüber informiert werden, damit sie sich gegebenenfalls für einen Wechsel an diese Einrichtung entscheiden können. Bei der Suche nach Alternativen hat das ärztliche Personal die Betroffenen nach Kräften zu unterstützen und soll allenfalls sogar zur Nutzung der Alternative raten, dies insbesondere dann, wenn das Abwarten erhebliche gesundheitliche Risiken birgt. Verletzen die Ärztinnen und Ärzte diese Pflichten, kann dies Haftungsfolgen nach sich ziehen. Erfolgt kein Wechsel und wird der Eingriff vielmehr am gleichen Spital verzögert durchgeführt, so sollte dies in der Patientenakte dokumentiert und begründet werden.

\section{Unsorgfältige Behandlung wegen Ressourcenknappheit?}

Mit einer Reduktion der Operationskapazitäten zugunsten von Covid-Erkrankten räumt das Spital der unmittelbaren Lebensrettung Vorrang ein, noch vor der Rettung möglichst vieler Lebensjahre und der Erhaltung bzw. Verbesserung der Lebensqualität. Dies lässt sich, wie erwähnt, aus Sicht der Grundrechte auf 
institutioneller Ebene rechtfertigen. Man wird dem Spital jedenfalls nicht zum Vorwurf machen können, dass es derzeit an einem demokratisch legitimierten Gesetz fehlt, das die Kriterien für die Zuteilung knapper Ressourcen verbindlich festlegt. Ebenso wenig haben die Spitäler nach aktueller Rechtslage eine Pflicht zur Aufrechterhaltung bestimmter Opera-

\section{Es fehlt an einem demokratisch legitimier- ten Gesetz, das die Kriterien für die Zuteilung knapper Ressourcen verbindlich festlegt.}

tionskapazitäten für planbare Eingriffe. Die Zuteilung knapper Ressourcen darf in den Spitälern jedoch nicht auf unsachlicher Entscheidung beruhen und bspw. zur Bevorzugung eines privatversicherten Patienten mit einer wenig dringlichen Operation gegenüber einer allgemeinversicherten Patientin mit einer dringlicheren Operation führen. Wenn ein Spital mit einem Patienten oder einer Patientin bereits einen Spitalaufnahmevertrag abgeschlossen hat, ist es aufgrund seiner möglichen Alternativen zu unterstützen. Wird dies unterlassen, stellt die Verletzung der Treuepflicht wiederum einen möglichen Haftungsgrund dar.

Prof. Dr. iur.

Bernhard Rütsche

Frohburgstrasse 3

CH-6000 Luzern

Tel. 0412295369

bernhard.ruetsche[at]

unilu.ch

\section{Das Wichtigste in Kürze}

- Im Herbst 2020 haben zahlreiche Spitäler über mehrere Monate hinweg ihre Operationskapazitäten gezielt und deutlich reduziert und die gewonnenen Ressourcen zugunsten von Covid-Erkrankten eingesetzt.

- Mit dieser Ressourcenreallokation wurde der unmittelbaren rungsentscheidung ist zwar aus grundrechtlicher Sicht vertretbar, aber nicht hinreichend demokratisch legitimiert.

- Ärztinnen und Ärzte sind in der Pflicht, die von verschobenen Operationen Betroffenen hinreichend über die Konsequenzen der Verschiebung und über mögliche Alternativen zu informieren. Treuepflicht zudem gehalten, diese bei der Suche nach

Eine andere Situation liegt vor, wenn aufgrund ausgedünnter oder fehlender Ressourcen eine Behandlung nicht nach den üblichen Standards durchgeführt werden kann. Kommt es in solchen Fällen zu einem Schaden, werden sich Arzt oder Ärztin und Spital ggf. mit dem Beweis entlasten können, dass sie die Krisensituation nicht verschuldet und immer noch ihr Bestes gegeben bzw. alles getan haben, was ihnen in der Krisensituation zumutbar war.

\section{Bildnachweis}

(c) Sudok1| Dreamstime.com

\section{Referenzen}

Dieser Artikel beruht auf der juristischen Fachpublikation von Bernhard Rütsche/Walter Fellmann/Regina E. Aebi-Müller, Verschiebung planbarer Spitalbehandlungen bei Ressourcenknappheit, Jusletter vom 19. April 2021.

\section{Literatur}

1 Kritisch zum Begriff der «elektiven» Operation auch NZZ vom 19. November 2020, Welche Operationen die Spitäler jetzt wegen Corona verschieben, abrufbar unter https://www.nzz.ch/schweiz/ welche-operationen-die-spitaeler-wegen-corona-verschiebenld.1587601.

2 Bundesamt für Gesundheit, Influenza-Pandemieplan Schweiz Strategien und Massnahmen zur Vorbereitung auf eine Influenza-Pandemie, 5. Auflage 2018, S. 95, 97 f.

\section{L'essentiel en bref} Lebensrettung oberste Priorität eingeräumt. Diese Priorisie-
- A l'automne 2020, de nombreux hôpitaux ont réduit considérablement et de manière ciblée leurs capacités chirurgicales. Les ressources obtenues ont été utilisées pour traiter les personnes atteintes de Covid.

- $\quad$ Avec cette réaffectation des ressources, la priorité absolue a été donnée pour sauver des vies. Si cette décision de priorisation est défendable du point de vue des droits fondamentaux elle ne bénéficie pas d'une légitimité démocratique suffisante.

- Les médecins ont le devoir d'informer de manière adéquate les personnes concernées par le report d'une opération sur les conséquences de ce report et sur les alternatives possibles. 